\title{
Auto-exame de mama: significado para pacientes em tratamento Quimioterápico
}

\author{
Breast self examination: meanins for patients in chemoteherapeutic treatment \\ Autoexamen de mamas: significado para pacientes en tratamiento Quimioterapico
}

\author{
Talita Garcia do Nascimento', Sueli Riul da Silva', Ana Rita Marinho Machado' \\ 'Universidade Federal do Triângulo Mineiro. Curso de Graduação em Enfermagem. Uberaba, MG
}

Submissão: $05 / 09 / 2008$

Aprovação: 22/07/2009

\section{RESUMO}

Verificar a prática e identificar o significado do auto exame de mamas entre portadoras de câncer submetidas à Quimioterapia foram objetivos desse trabalho. Tratou-se de pesquisa Qualitativa, utilizando o discurso de portadoras de câncer de mama em Quimioterapia no Hospital de Clínicas da Universidade Federal do Triângulo Mineiro, através de entrevista semi-estruturada. O material coletado foi submetido à análise de conteúdo simplificada, proposta por Thiollent. As entrevistadas possuíam conhecimento sobre a existência e importância do AEM, porém, muitas não o praticavam de forma correta ou desconheciam a periodicidade e técnica indicada, havendo necessidade de desenvolver intervenções efetivas Que promovam a conscientização da população feminina a respeito da importância em adotar práticas direcionadas ao diagnóstico precoce do câncer mamário.

Descritores: Câncer de mama; Auto-exame de mama; Enfermagem.

\begin{abstract}
To verify the practice and to identify the meaning of the breast self examination among women with cancer and were submitted to the chemotherapy were objectives of this work. It was a Qualitative research, using the speech of the women who are breast cancer carriers in the Hospital das Clínicas of the Universidade Federal do Triângulo Mineiro, Uberaba, MG, Brazil through semi-structured interviews. The collected material was submitted to the analysis of the simplified content proposed by Thiollent. The interviewees owned knowledge on the existence and Importance of breast self examination, however, many did not practice it correctly or ignored the periodicity and technic indicated, there being, this way, need to develop effective interventions to promote the understanding of the feminine population concerning the importance in adopting practices aimed at the precocious diagnosis of the mammary cancer.
\end{abstract}

Descriptors: Breast neoplasms; Breast self examination; nursing.

\section{RESUMEN}

Verificar la práctica y identificar el significado del auto examen de mamas (AEM) entre portadoras de cáncer sometidas a la Quimioterapia fueron objetivos de ese trabajo. Se trató de pesquisa cualitativa, utilizando el discurso de portadoras de cáncer de mama en QT en el Hospital de Clínicas de la Universidade Federal do Triângulo Mineiro, través de entrevista séme-estructurada. El material colectado fue sometido a la analice de contenido sencilla, propuesta por Thiollent. Las entrevistadas poseían conocimiento sobre la existencia y importancia del autoexamen de mamas, pero, muchas no lo practicaban de manera correcta o desconocían la periodicidad y técnica indicada, habiendo necesidad de desarrollar intervenciones efectivas Que promuevan la consciencia de la población femenina a respecto de la importancia en adoptar prácticas dirigidas al diagnóstico precose del cáncer mamario.

Descriptores: Cáncer de mama; Autoexamen de mama; Enfermería. 


\section{INTRODUÇÃO}

A assistência médica social e coletiva deve ser praticada com base em dois pontos principais: prevenção e diagnóstico efetivo das diferentes doenças que acometem o ser humano, principalmente no caso de enfermidades com alto índice de mortalidade, como as doenças cardiovasculares e o câncer. No caso do câncer de mama, Que é provavelmente o mais temido pelas mulheres devido à sua alta freqüência e, sobretudo pelos seus efeitos psicológicos Que afetam a percepção da sexualidade e a própria imagem pessoal, alguns pesquisadores acreditam Que só será possível reduzir sua mortalidade com diagnóstico precoce Que identifieue o tumor com o menor tamanho possível em estádio inicial ${ }^{(1,2)}$.

O câncer de mama caracteriza-se como a principal neoplasia maligna Que acomete o sexo feminino no Brasil, apresentando a taxa bruta de incidência estimada, para 2006, de 48.930, com um risco estimado de 52 casos a cada 100 mil mulheres. Na região Sudeste é o mais incidente com um risco estimado de 71 casos novos por 100 mil. Sem considerar os tumores de pele não melanoma, este tipo de câncer também é o mais freQüente nas mulheres das regiões Sul (69 casos por 100.000 mulheres), CentroOeste (38 casos por 100.000 mulheres) e Nordeste (27 casos por 100.000 mulheres). Na região Norte é o segundo tumor mais incidente ( 15 casos por 100.000 mulheres) $)^{(1,3)}$.

Considerando a origem dos cânceres mamários, acredita-se Que $90 \%$ a $95 \%$ deles sejam esporádicos (não familiares) e decorram de mutações somáticas, Que se verificam durante a vida, e Que 5\% a 10\% sejam hereditários (familiares), devido a mutações nucleotídicas perpetuadas na linhagem familiar pelas células germinativas, Que confere suscetibilidade ao câncer de mama. Considerando ainda Que o processo carcinogênico deva ser conduzido por mutações gênicas, existem muitas variáveis epigenéticas, particularmente aQuelas relacionadas com ações autócrinas, parácrinas e endócrinas de moléculas reguladoras ${ }^{(4)}$.

O tratamento do câncer pode ser realizado basicamente por Quatro abordagens: a cirurgia (mastectomia unilateral, bilateral, total ou parcial) e radioterapia, como tratamentos locais; a Quimioterapia e a terapia com agentes biológicos (como hormônios, anticorpos ou fatores de crescimento) como tratamentos sistêmicos ${ }^{(5)}$.

As formas mais eficazes de detecção precoce do câncer de mama são: o exame sistemático da mama, ou exame clínico, feito pelo profissional especializado; a mamografia, Que consiste em um exame radiológico de alta precisão e custo igualmente elevado, o Que dificulta o acesso da população de menor poder aQuisitivo; e para finalizar, o auto exame das mamas (AEM), caracterizado pela facilidade e baixo custo, já Que Quem o executa é a própria mulher. A mamografia e ultra-sonografia identificam tumores não palpáveis, apresentam alto custo e não fornecem resultados operacionais para serem aplicados em grandes massas populacionais, consagrandose o AEM mensal como estratégia de escolha, uma vez Que se caracteriza como prevenção secundária, sem custos e segura(6,7).

De acordo com pesquisas realizadas, foi evidenciado um impacto significativo do AEM na detecção precoce do câncer de mama, registrando-se tumores primários menores e menor número de linfonodos axilares invadidos pelo tumor (ou por células neoplásicas) nas mulheres Que fazem o exame regularmente, além de haver também detecção de peQuenas mudanças nas propriedades físicas das mamas, diminuindo assim a probabilidade de metástase e aumentando a sobrevida dessas pacientes ${ }^{(6)}$.

Embora o conhecimento das vantagens do auto-exame das mamas seja bastante divulgado pelos meios de comunicação, setores educacionais e programas de saúde pública, diversos estudos demonstram que o índice de prática é incipiente em diversas partes do mundo. Demais pesquisas sobre fatores sócio-econômicos Que influenciam a prática do exame, mostram Que as mulheres de maior nível educacional e renda são as de maior adesão e Que detêm maiores conhecimentos sobre este, em contrapartida, foi verificado Que as camadas mais carentes de informação e conscientização sobre a importância dessa técnica na detecção precoce do câncer de mama apresentam alta taxa e desconhecimento e não o praticam $^{(7,8)}$.

Como a ocorrência do câncer de mama é uma possibilidade na vida de toda mulher, decorre desses argumentos a relevância do presente estudo, Que busca identificar entre mulheres portadoras de câncer de mama o significado atribuído ao AEM.

\section{OBJETIVOS}

Verificar a prática do AEM entre pacientes portadoras de câncer de mama submetidas à Quimioterapia.

Identificar o significado atribuído ao AEM entre mulheres portadoras de câncer de mama.

\section{METODOLOGIA}

O presente estudo foi desenvolvido através de uma pesquisa Qualitativa, por meio do discurso de mulheres portadoras de câncer de mama a respeito da freqüência da prática do auto exame e o significado dado a este por esta população. Foram entrevistadas 10 mulheres portadoras de câncer de mama durante as seções de Quimioterapia na Enfermaria de Ginecologia e Obstetrícia do Hospital de Clínicas da Universidade Federal do Triângulo MineiroUFTM e que se mostraram dispostas a participarem do estudo. Foram incluídas todas as pacientes maiores de 18 anos, sem restrição de etnia, escolaridade ou classe social e, somente não participaram do estudo aquelas Que não concordaram ou não atenderam os critérios acima descritos.

Os dados foram coletados no período de novembro de 2007 a março de 2008, após aprovação do projeto pelo Comitê de Ética em Pesquisa da UFTM (Protocolo 956/07). O primeiro contato foi realizado na própria enfermaria após ter iniciado as seções de Quimioterapia, onde a paciente recebeu os esclarecimentos pertinentes Quanto o estudo a ser realizado concomitante aos seus objetivos. Após o termo de esclarecimento ter sido lido e o de consentimento assinado, deu-se início a coleta dos dados propriamente dita.

A técnica utilizada para a coleta de dados foi baseada em uma entrevista semi-estruturada. Esta consiste em um instrumento que combina Questões abertas relacionadas com os problemas Que foram considerados relevantes e desencadeia uma série de perguntas que permite um progressivo aprofundamento dos problemas. A abertura das perguntas visa deixar aos entrevistados uma total liberdade de apreensão da realidade ${ }^{(9)}$.

Para realização das entrevistas utilizou-se um roteiro com 
Questões pré-estabelecidas. O roteiro foi dividido em duas partes: a primeira continha dados de identificação, tais como o codinome escolhido pela paciente, idade, procedência, profissão/ocupação e estado civil e, a segunda as Questões norteadoras do estudo. Foi utilizado registro manual das informações.

O material coletado foi submetido à análise de conteúdo simplificada, proposta por Thiollent ${ }^{(9)}$, de modo a listar as principais informações contidas nas entrevistas. A análise temática é principalmente Qualitativa e busca identificar os termos mais importantes do ponto de vista dos atores e as várias expressões nas Quais são utilizados. Seu objetivo é descobrir as significações dos termos Que são utilizados espontaneamente pelos participantes e relacionados com temas julgados relevantes para mapear os problemas encontrados na situação a ser investigada ${ }^{(9)}$.

A análise temática foi iniciada pela busca de palavras-chave e de seus derivados na transcrição das entrevistas individuais. Em seguida, os trechos foram depurados e classificados "de modo a evidenciar as características do tratamento Que os temas recebem no discurso dos entrevistados"(9).

\section{RESULTADOS E DISCUSSÃO}

O Quadro I apresenta as principais características de identificação das mulheres entrevistadas.

Através da análise dos dados foi possível identificar Que as entrevistadas possuíam o conhecimento sobre a existência do auto exame das mamas, porém, muitas não o praticavam de forma correta ou, até mesmo desconheciam a periodicidade e a técnica indicada para sua realização. Foi observado Que mesmo conhecendo o AEM, esse não se encontrava incluído nas práticas de prevenção contra o câncer de mama na rotina de algumas mulheres e só se fez presente após o aparecimento da doença, como demonstram as falas a seguir:

Fazia, mas nunca tinha visto (o nódulo). A menstruação sumiu e o seio tava inchado, eu Que senti o caroço (Rosa).

Fazia, mas não sentia nada, só ficava vermelho (Palma).

Sim, foi aí Que eu descobri, porQue senão...(Violeta).

Faço (Oreuídea).

Sim (Estreliça)
Faço (Bromélia).

Antes não. Já tinha ouvido falar, vê nos outros mas não na gente (Tulipa).

Fazia não todos os dias, mas fazia. Quando eu lembrava eu fazia, as vezes deitada ou Quando ia tomar banho (Petúnia).

Não tinha esse costume, lerdeza. Eu conhecia só que sei lá.

Mais engraçado, nunca parei para fazer direitinho, como manda o figurino (Gérbera).

Eu fazia de vez em Quando, mas não muito bem. Esse (exame) eu só fazia de vez em Quando, não dava muita bola não. Eu só mexia Quando tava tomando banho, debaixo da água. Agente descuida agora agente faz (Hortência).

Partindo do pressuposto Que indivíduos praticantes do AEM são considerados somente aQueles Que referem fazê-lo de forma regular, ou seja, mensalmente ${ }^{(8)}$, através das falas das entrevistadas foi possível destacar que grande parte delas conhecia o exame, porém poucas o praticavam ou praticavam da maneira incorreta. Tal dado é confirmado por demais pesquisas Que evidenciam Que ao mesmo tempo em Que $95,3 \%$ das mulheres referem conhecer o AEM, destas apenas 7,4\% possuem conhecimento adequado para esse procedimento ${ }^{(2)}$.

Vários aspectos têm sido peseuisados sobre as causas para a não realização ou a realização incorreta do AEM. Um aspecto frequentemente mencionado diz respeito àqueles de natureza cultural envolvendo sua prática. Estudo realizado no Canadá entre diferentes grupos étnicos revelou Que a resistência à prática do AEM, ao exame clínico das mamas e à realização da mamografia é mais elevada entre populações nativas (indígenas) Que nas comunidades de origem ucraniana, finlandesa e italiana. Por outro lado, existem relatos de Que, entre grupos com acesso pleno a informações correlatas, a prática do AEM não apresenta diferenças entre mulheres de diferentes bagagens culturais ${ }^{(8)}$. Outros autores afirmam Que o principal motivo da não-realização é o desconhecimento da técnica, seguido por esquecimento, o Que se justifica pelo fato de Que a maioria dos serviços Que realizam atendimento em saúde da mulher apresenta propostas Que não são

\begin{tabular}{|c|c|c|c|c|c|}
\hline Participantes & Idade & Estado Civil & Escolaridade & Profissão/ Ocupação & Procedência \\
\hline Rosa & 44 & solteira & $2^{\circ}$ grau comp. & auxiliar de nutrição & Uberaba/ MG \\
\hline Palma & 65 & casada & magistério & do lar & Uberaba/ MG \\
\hline Violeta & 45 & casada & $l^{\circ}$ grau incomp. & do lar & Catalão/ GO \\
\hline Oreuídea & 66 & casada & não freeüentou escola & do lar & Sacramento/ MG \\
\hline Estreliça & 43 & separada & $2^{\circ}$ grau comp. & autônoma & Uberaba/ MG \\
\hline Bromélia & 33 & casada & $l^{\circ}$ grau incomp. & do lar & Uberaba/ MG \\
\hline Tulipa & 38 & solteira & $1^{\circ}$ grau incomp. & auxiliar de enfermagem & Sacramento/ MG \\
\hline Petúnia & 60 & casada & $2^{\circ}$ grau comp. & aposentada & Uberaba \\
\hline Gérbera & 30 & casada & magistério & do lar & Compos Altos/MG \\
\hline Hortência & 62 & viúva & $l^{\circ}$ grau incomp. & do lar & Sacramento/ MG \\
\hline
\end{tabular}

Quadro 1. Identificação das mulheres entrevistadas. 
acompanhadas de treinamento adequado para a prática do AEM. Esse mesmo estudo evidenciou também Que aspectos importantes para a não-realização são justificados em "não achar necessário" e a "falta de interesse", demonstrando Que apenas transmitir informação não é suficiente para mudança de comportamento, já Que a prática do AEM depende da decisão da cliente, a partir da compreensão e interpretação Que tem da possibilidade de prevenir e ser responsável pela sua própria saúde ${ }^{(6)}$.

A freeüência na realização do AEM influencia diretamente a eficácia do mesmo. Segundo estudos, para mulheres Que nunca praticaram o exame, geralmente os nódulos cancerígenos identificados medem $3,5 \mathrm{~cm}$, para as Que o praticam eventualmente, os nódulos têm cerca de $2,5 \mathrm{~cm}$; e, para as Que o fazem mensalmente, são identificados com aproximadamente $2 \mathrm{~cm}$ ou menos. Aquelas que praticam o AEM e descobrem os nódulos, têm expectativa de vida de $75 \%$ e as Que não o fazem reduzem suas chances para $59 \%$.

Diante da constatação ou não da prática do AEM, bem como o seu conhecimento pelas pacientes portadoras de câncer de mama, essas mulheres foram Questionadas Quanto ao significado dessa prática. Assim, de acordo com as entrevistadas, o AEM apresenta principalmente sinônimo de prevenção e cuidado com a própria saúde:

Significa eu tentar saber se tem algum problema comigo (Rosa).

Uai acho ele muito importante porque e só com ele que pode detectar se tem alguma coisa Que não ta normal. Acho ele muito importante (Violeta).

Uai, isso e pro bem da gente mesmo (OrQuídea).

Uma prevenção porQue eu posso diagnosticar antes (Estreliça).

Ah, acho Que é prevenção mesmo, eu estou me prevenindo (Bromélia).

Significa vida. Se parar com o tratamento volta tudo de novo (Tulipa).

Ah, sei lá. Olhando hoje acho muito importante. Não sei, acho Que se eu tivesse visto antes, apalpado...(Gérbera).

Estudos anteriores vêm de encontro aos relatos apresentados pelas entrevistadas acima, onde estas reconhecem a prática do AEM como uma forma de prevenção do câncer de mama. De acordo com outros autores, crenças e comportamentos modais salientes relativos ao comportamento interferem no significado do AEM entre as pacientes e através deste, estão representados suas vantagens e desvantagens na utilização de tal prática ${ }^{(7)}$. Foram avaliadas individualmente categorias de crenças comportamentais Que sugeriram a significância do AEM como: "não haver nenhuma desvantagem na não realização do AEM" (resposta favorecida possivelmente pelo baixo nível de informação e escolaridade das participantes do estudo), "encontrar nódulos" (relaciona-se com as mensagens veiculadas pela mídia geralmente alertando para esta vantagem do exame), "evitar o câncer de mama" (vai ao mesmo sentido da anterior), categoria "cuidar da saúde”, "confiança médica" (respalda-se no papel do médico como detentor de conhecimentos e habilidades relacionados à saúde, sendo pois, caracterizada como uma crença negativa, uma vez Que enQuanto a mulher prefere Que o médico a examine, ela não desempenha o comportamento, podendo não detectar o câncer precocemente), "temor a doença" (implica no estigma da doença como sinônimo de morte), "evitar hospitais" (relacionada com o contexto do sistema público de saúde brasileiro, com filas enormes, demora em marcar consultas etc) ${ }^{(7)}$.

Considerando as categorias "encontrar nódulos" e "evitar o câncer de mama" é possível fazer uma análise do poder exercido pelos meios de comunicação de massa na vida da população. Alguns autores afirmam ser o serviço de saúde e os meios de comunicação em massa os maiores disseminadores do conhecimento e do ensino da prática do AEM. A orientação pela imprensa atingiu grande número de mulheres, porém, a informação fornecida não se mostrou eficiente por não ensinar a prática correta do exame, visto Que grande parte das mulheres incluídas no estudo realizava o exame com freeüência incorreta. Campanhas Que atingem a maior parte da população não orientam a prática de forma adęuada, além de não haver programas eficientes de ensino do AEM em serviços de atendimento a mulher. É importante Que a detecção precoce do câncer de mama por meio do ensino do auto exame seja de responsabilidade de todos os Que assistem pacientes do sexo feminino, e não apenas daQueles Que atuam em programas específicos para esse fim $^{(6)}$

O caráter sombrio e imprevisível do câncer, particularmente o câncer de mama, pode ser facilmente evidenciado em vários estudos, Quando as mulheres pesquisadas fazem comparações com outras enfermidades durante seu processo de significação. O câncer é comparado à AIDS Que, na atualidade, é uma doença com significações éticas, morais e sexuais predominantemente mais repressivas. Com essa interpretação, os depoimentos das mulheres estudadas apontam a AIDS como possível de ser evitada, enQuanto Que o câncer ainda não. Simbolicamente, as imagens suscitadas tanto pela AIDS como pelo câncer revelam o seu caráter de finitude. Nesse processo de significação, onde o futuro é incerto, parece Que a morte é iminente ${ }^{(10)}$.

Dado o caráter de finitude da vida frente ao câncer pelas mulheres que o possui, o AEM reflete de forma oposta para essa população, trazendo consigo o significado de vida, prevenção, auto cuidado e até mesmo, esperança de cura.

Buscando o significado preventivo da prática do AEM e sua importância, as pacientes foram Questionadas sobre o incentivo Que dariam às demais mulheres sobre a utilização da técnica em suas vidas. Por unanimidade, todas afirmaram a importância de disseminar a utilização do AEM nas mais diferentes classes femininas.

Sim, agente faz que é preciso. Se agente se cuidar mais antes é melhor pra não deixar a doença tomar conta (OrQuídea).

Nossa! Hoje incentivo muito mais. Só para todas que eu conheço, para não passar por tudo isso (Estreliça).

Incentivaria (Bromélia).

Incentivaria, porQue é uma coisa Que se não cuidar a tempo fica 
mais difícil de tratar (Tulipa).

Eu incentivaria sim, porque é importante, mesmo que eu não percebi, ainda acho importante (Petúnia).

Ah, hoje eu vejo a importância dele. Hoje eu falo pra minha cunhada: você fez? Antes parece Que agente fica meio isolada, depois Que acontece agente vai atrás de um tanto de informação (Gérbera).

Durante o processo de adoecer e, evidenciado aQui, o câncer de mama, as mulheres buscam identificar sentimentos e para tal tentam compreender sua própria responsabilidade nesse processo, assim passam a conviver com uma nova concepção de si mesmas, a Qual nem sempre é fácil de ser aceita. Assim tendem a se comportar como pessoas com um estigma particular Que tendem a vivenciar experiências similares relativas à aprendizagem ${ }^{(10)}$. É, pois, nesse sentido Que o incentivo a práticas de prevenção como o AEM, passam a ser parte de seus repertórios, a fim de sensibilizar as demais mulheres pertencentes ou não a seus círculos de convivência, para evitar Que estas não "passem por tudo isso".

O câncer de mama traz à consciência das mulheres a importância do cuidado consigo mesmas, e as repercussões Quando é colocado em segundo plano. Diante da situação vivida, muitas passam a refletir acerca das ações tomadas com relação à sua saúde e percebem o descaso Que tiveram com o próprio corpo. As mulheres através de seus depoimentos deixam transparecer sentimentos de culpa decorrentes da falta de cuidado consigo mesmas ${ }^{(10)} \mathrm{e}$ demonstram a importância de se manter atenta Quanto as alterações percebidas no próprio corpo e dão ênfase no incentivo de tais práticas às mulheres Que não se encontram em situação semelhante.

\section{CONSIDERAÇÕES FINAIS}

A discussão feita através dos dados colhidos sobre o significado do AEM pelo grupo de pacientes portadoras de câncer de mama em QT, evidencia a necessidade do desenvolvimento de intervenções efetivas direcionadas à assistência integral tanto para aQuelas atingidas pelo estudo Quanto para as demais mulheres, pertencentes as mais variadas faixas etárias.

O conhecimento e a conotação preventiva dada ao AEM se fazem presentes em todos os discursos registrados, porém, a utilização correta da técnica e a fręüência em realizá-la seguem caminhos opostos.

Grande parte dos meios de comunicação em massa superestima a utilização desse método, como sendo o único ou o mais importante na luta contra o câncer de mama, esquecendo, pois, de informar a população como e Quando deve ser realizado e excluindo de seu discurso as demais práticas preventivas como a mamografia, o exame clínico das mamas e a radiografia.

É nesse contexto Que se faz necessária a implementação de estratégias eficazes Que incentivem não somente a utilização correta do auto exame, como também os demais métodos preventivos e, principalmente a conscientização dessa população da necessidade em adotar práticas direcionadas ao diagnóstico precoce do câncer mamário.

\section{REFERÊNCIAS}

I. Ministério da Saúde (BR). Secretaria Nacional de Assistência à Saúde. Instituto Nacional do Câncer. Estimativas de incidência e mortalidade por câncer no Brasil. Rio de Janeiro: INCA; 2006.

2. Marinho LAB, Gurgel MSC, Cecatti JG, Osis MID. Conhecimento, atitude e prática do auto-exame das mamas em centros de saúde. Rev Saúde Pública 2003; 37(5): 576-82.

3. Sclowitz ML, Menezes AMB, Gigante DP, Tessaro S. Condutas na prevenção secundária do câncer de mama e fatores associados. Rev Saúde Pública 2005; 39(3): 340-9.

4. Oliveira VM, Aldrighi JM, Rinaldi JF. Quimioprevenção do câncer de mama. Rev Assoc Med Bras 2006; 52(6): 453-9.

5. Anjos ACY, Zago MMF. A experiência da terapêutica Quimioterápica oncológica na visão do paciente. Rev LatinoAm Enfermagem 2006; I 4(I): 33-40.

6. Monteiro AAPS, Arraes EPP, Pontes LB, Campos MSS, Ribeiro
RT, Gonçalves REB. Auto-exame das mamas: freeüência do conhecimento, prática e fatores associados. RBGO 2003; 25(3): 201-5.

7. Gonçalves SCM, Dias MR. A prática do auto-exame da mama em mulheres de baixa renda; um estudo de crenças. Estudos Psicol 1999; 4(1): 141-59.

8. Junior RF, Koifman S, Santos NRM, Nunes MOA, Melo GG, Ribeiro ACG, Melo AFB. Conhecimento e prática do auto-exame de mama. Rev Assoc Med Bras 2006; 52(5): 337-41.

9. Thiollent M. Pesquisa --Ação nas organizações. São Paulo: Atlas; 1997.

10. Almeida AM, Mamede MV, Panobianco MS, Prado MAS, Clapis MJ. Construindo o significado da recorrência da doença: a experiência de mulheres com câncer de mama. Rev Latino-Am Enfermagem 2001; 9(5): 63-9. 